\title{
Editorial
}

\section{Heart rate variability and clinical cardiology}

The first observation that heart rate variability (HRV) could be used as a predictor of mortality after acute myocardial infarction was published in $1978 .{ }^{1}$ Since then the usefulness of HRV for the identification of patients at high risk after acute infarction has been confirmed in large studies. ${ }^{23}$ Not surprisingly, these discoveries led to widespread interest in HRV among clinical investigators and until now HRV has been not only the subject of many physiologically oriented investigations but also its potential practical value has been assessed in patients from many different clinical groups.

There are important differences between the type of heart rhythm recordings used for purely physiological studies and those used for fully clinical studies. Whereas the physiologist tends to obtain recordings of heart rhythm under strictly controlled conditions, the clinician generally uses standard ambulatory electrocardiograms, and data from these are much less stationary. In clinical recordings the different processes modulating the heart rate are much less easily distinguishable.

The editorial by Malliani et al on page 1 of this issue 4 describes the state-of-the-art of the physiological background of HRV. We will discuss contemporary experience with and understanding of the clinical use of HRV. Although assessment of HRV is of indisputable value in many non-cardiological clinical disciplines-for example, neurology, ${ }^{5}$ diabetology, ${ }^{6}$ obstetrics, ${ }^{7}$ occupational medicine ${ }^{8}$ - we will concentrate solely on the potential use of HRV in clinical cardiology.

\section{Cardiac failure}

HRV is depressed in patients with heart failure. However, these patients have faster heart rates, owing to the predominance of sympathetic tone, which partly accounts for the depression of HRV. In addition, maintaining a minimum diastolic interval ${ }^{9}$ tends to reduce the variation of heart periods even more in patients in heart failure. Several studies have been conducted to investigate the sympathetic and parasympathetic components of HRV in heart failure. Coumel et al investigated the effects of medium-term $\beta$ blockade and found that heart rate was significantly more depressed in patients with heart failure rate than in healthy controls. ${ }^{10}$ They also found a specific decrease in the sympathetic components of HRV in heart failure. ${ }^{10} \beta$ blockade can depress all the HRV components in heart failure. Depending on the status of the autonomic system.

Nolan et al specifically investigated the parasympathetic measures of HRV in chronic heart failure and observed a strong correlation between these measures and left ventricular ejection fraction. ${ }^{11}$ This may suggest that the degree of cardiac parasympathetic malfunction is related to the severity of left ventricular malfunction. However, this observation was not confirmed by Kienzle et al who correlated different spectral components of HRV with various clinical variables and did not find any significant correlation between HRV and left ventricular ejection fraction, New York Heart Association (NYHA) functional classification, or age. ${ }^{12}$ Despite this, both high and especially low frequency components of HRV were related to cardiac output.

Stein et al reported a link between reduced HRV and adverse events in patients with severe mitral regurgitation. ${ }^{13}$ In their study only the measures of ultra-low frequencies of HRV were correlated with left and right ventricular ejection fraction and these measures of HRV were most predictive of mortality, disease progression, and development of atrial fibrillation.

All spectral components of HRV have repeatedly been observed to be depressed in patients with heart failure but the pathophysiological mechanism remains unclear. At present the assessment of HRV is of no practical value in the management of patients with heart failure.

\section{Heart transplantation}

A recently transplanted heart has no innervation. Therefore the heart transplant recipient exhibits reduced HRV. Sands et al observed not only a reduced total spectral power of HRV in these patients but also power spectra of HRV without any distinct peaks. ${ }^{14}$ They also found that the total spectral power of HRV was increased in those patients with transplants in whom allograft rejection developed but not in those whose endomyocardial biopsy specimens showed no evidence of rejection. In patients with rejection, however, HRV was increased by overall chaotic irregularities of the cardiac rhythm without noticeable periodic components.

Bernardi et al observed depressed HRV at rest in patients with transplants but found direct correlation between the high frequency components of $\mathrm{HRV}$ and respiration rate during exercise. ${ }^{15}$ This suggests that a non-autonomic nervous mechanism, possibly humoral or intrinsic to the myocardium but masked by autonomic effects in non-transplanted hearts, influences HRV at peak exercise in synchrony with ventilation. Smith et al studied HRV changes caused by reinnervation of the transplanted heart. ${ }^{16}$ They reported that parasympathetic components of HRV do not rise during the first month after transplantation but increase significantly between 3 and 6 months, suggesting early reinnervation of the parasympathetic system.

Thus the reduction of total values of HRV as well as the disappearance of its distinct spectral peaks in cardiac transplant recipients is well established. The observation that a white-noise-like increase of HRV might be a warning sign of allograft rejection needs further confirmation. Reinnervation is likely to be detected by the reappearance of physiological spectra rather than by an increase in the total power of HRV in transplanted patients.

\section{Essential hypertension}

Guzetti et al studied the effect on HRV of enhanced sympathetic activity in patients with essential hypertension. ${ }^{17}$ 
Compared with normotensive controls the low frequency components of HRV were larger in hypertensive patients and the high frequency components were smaller. A passive tilt produced smaller changes in the relation between the high and low frequency components of HRV in hypertensive patients than in controls. Moreover, both the values of low frequency components and the extent of the altered effects of tilt on HRV components strongly correlated with the degree of hypertension. Chronic $\beta$ adrenergic blockade which reduced blood pressure in hypertensive patients also changed the HRV components towards normal values. Thus measurement of HRV might assess progressive alterations in the sympathovagal balance observed in essential hypertension. No study, however, has so far demonstrated a strong correlation between HRV variables and cardiac arrhythmias or the risk of sudden death in essential hypertension.

\section{Risk stratification after myocardial infarction}

The most updated use of HRV is for risk stratification after myocardial infarction. Wolf et al first reported that patients with acute myocardial infarction who lack respiratory sinus arrhythmia have a significantly higher risk of in-hospital mortality. ${ }^{1}$ Later, this observation was confirmed for the post-discharge phase by several centres using independent data. Other recent studies have reproduced the original findings of Wolf et al and, in addition, have shown that HRV assessed during the acute phase of myocardial infarction is significantly related to clinical and haemodynamic indices of severity, such as peak creatine kinase, left ventricular ejection fraction, and Killip class. ${ }^{18}$

Compelling evidence that $\mathrm{HRV}$ is a powerful risk predictor after myocardial infarction first came from the investigators of the Multicenter Post-Infarction Research Group. In their report, Kleiger et al showed a strong association between depressed HRV and increased mortality during the first four years after acute infarction. ${ }^{2}$ By clustering the total population according to mean heart rate, left ventricular ejection fraction, frequency of ventricular extrasystoles, and presence of couplets or runs of ventricular extrasystoles Kleiger et al showed that depressed HRV predicted mortality independently of these recognised risk factors. In a subsequent study that investigated separately parasympathetic and sympathetic time domain HRV measures Bigger et al found significantly reduced parasympathetic activity in patients at risk of mortality after myocardial infarction. ${ }^{19}$ These findings were confirmed by other centres-for example, by the Post-Infarction Survey Programme at our own institution which showed that depressed HRV was not only capable of predicting the total cardiac mortality after infarction but also identified patients at risk of sustained symptomatic ventricular tachycardia. ${ }^{20}$ Recently, a large epidemiological study showed that reduced HRV and especially a reduction in its high frequency components is associated with an increased risk of sudden death in patients referred for Holter monitoring. ${ }^{21}$

Though substantial advances have been made, the pathophysiological link between depressed HRV and cardiac mortality is still unknown. In 1976, Lown and Verrier reported that decreased vagal tone predisposed to ventricular fibrillation in animals with experimental myocardial infarction. ${ }^{22}$ Furthermore, increased sympathetic activity during experimental ischaemia or infarction promotes ventricular fibrillation in animal models. The opposite is also valid: increased parasympathetic tone or decreased sympathetic activity reduces vulnera- bility to ventricular fibrillation in laboratory animals. This accords with the finding that $\beta$ adrenergic blocking drugs reduce the likelihood of ventricular fibrillation after myocardial ischaemia or infarction.

Whether reduced HRV in high risk survivors of infarction is the result of generally decreased autonomic tone or whether it is the consequence of diminished cardiac response to autonomic stimuli remains to be elucidated. Nevertheless, animal models have shown that the change in cardiac autonomic tone that makes the heart of some animals more susceptible to ventricular fibrillation occurs in close relation to the experimental infarction. Hull et al investigated $\mathrm{HRV}$ in dogs before and 1 month after experimental myocardial infarction. ${ }^{23}$ They did not find any difference in HRV assessed before infarction between those animals that survived 1 month and those that died suddenly during this period. They also observed that depression of HRV was much greater in those animals in which ventricular fibrillation developed during exercise and ischaemia.

Does the prognostic power of a post-infarction HRV value assessed from a short-term recording equal that measured from 24 hour electrocardiograms? Malik et al found that arbitrary short-term recordings are not sufficient because their specificity for predicting arrhythmia risk is small. ${ }^{24}$ Only when the total circadian variation of autonomic nervous activity was integrated did practically acceptable values of sensitivity and specificity emerge. However, we do not know the predictive power of HRV assessed from short-term recordings made under strict conditions-for example, controlled respiration. Several physiological studies have investigated such short-term recordings but the patient population has never been large enough to assess their predictive power. The observations made with arbitrary short-term recordings accord with a study by Bigger et al who compared the predictive power of different spectral components of HRV for the identification of the risk of post-infarction mortality. ${ }^{25}$ They found the highest predictive power in the ultra-low frequency components corresponding to heart rate oscillations in terms of hours rather than minutes.

These findings based on retrospective evaluations of large patient populations are not immediately linked to the pathophysiological experiments that suggest that depressed vagal activity is associated with arrhythmia risk. Under controlled laboratory conditions depressed vagal activity manifests itself as depressed high frequency HRV components. On the contrary, the retrospective clinical studies show that while the depressed "parasympathetic" HRV components have a predictive value, the risk of arrhythmic complications is more strongly associated with depressed ultra-low frequencies of HRV. The reasons for this discrepancy are poorly understood. It seems likely that the association of high and low frequency components of HRV with parasympathetic and sympathetic modulations of heart rate that has been clearly established in laboratory recordings ${ }^{4}$ may not be preserved in non-stationary ambulatory clinical recordings. Moreover, the spectral components of HRV reflect autonomically modulated variations of heart rate which, under pathological conditions, may not be directly linked to the autonomic tone itself. Even some technical problems-for example, incorrect assessment of HRV frequencies in high risk patients who have more frequent extrasystoles-cannot be excluded.

Odemuyiwa et al investigated whether post-infarction HRV is more powerful as a predictor of total cardiac mortality, of arrhythmic complications (that is, sudden deaths and sustained symptomatic ventricular tachycardia), or of sudden death alone. ${ }^{3}$ When the predictive 
powers of decreased HRV and decreased left ventricular ejection fraction were compared HRV was found to be about as powerful as the ejection fraction for predicting total cardiac mortality in the post-infarction population whereas it was significantly more powerful than the ejection fraction for predicting sudden death and symptomatic ventricular tachycardia. This finding accords with our understanding of the pathophysiology of depressed HRV after myocardial infarction. The reduced left ventricular ejection fraction is more likely to reflect a liability to cardiac failure and thus to identify patients who are at risk of non-sudden death, some of whom will also develop ventricular fibrillation and die suddenly. On the contrary, reduced HRV reflects the lack of an autonomic defence against ventricular fibrillation and thus more powerfully identifies those patients who are at high risk of sudden death or arrhythmic complications.

Investigations which have compared baseline values of HRV with those shortly preceding ventricular tachycardia or episodes of fibrillation are not fully conclusive. Generally depressed HRV has been observed in those who died suddenly ${ }^{26}$ and some studies have reported an increased ratio between low and high frequency components of HRV preceding ventricular tachycardia. ${ }^{27}$ Other studies, however, have not found any systematic changes of HRV preceding ventricular fibrillation. ${ }^{28}$ Thus it is not known whether the depressed HRV in post-infarction patients is a complex multifactorial marker of arrhythmia risk or whether it has a more direct mechanistic relation to the proarrhythmic substrate, triggers, and mechanisms. Mental concentration and psychological conditions are known to influence HRV. Therefore the psychosomatic correlates of cardiac morbidity may also play an important part in preserving or depressing HRV after acute infarction. Thus the association of depressed HRV with arrhythmia-related complications might just be an incidental mixture of many factors that make depressed HRV such a statistically significant but mechanistically immaterial factor. The fact that we do not fully understand the explanation for depressed HRV does not make its clinical importance less valid.

The combination of HRV with other risk factors seems to be particularly useful in risk stratification after acute myocardial infarction. Though only limited data have been published it seems that a combination of HRV, late potentials detected in the high gain electrocardiogram, and the frequency of ventricular extrasystoles is a very predictive combination. In such a setting, late potentials may reveal an anatomical substrate for mediating reentrant tachyarrhythmias: ventricular ectopic activity provides frequent triggers for arrhythmias and depressed HRV indicates diminished parasympathetic defence against ventricular fibrillation.

Another possible prognostic combination is HRV with baroreflex sensitivity. Though both $\mathrm{HRV}$ and baroreflex sensitivity assess autonomic function, the correlation between both measures is weak. ${ }^{29}$ This is probably because HRV reflects the physiological level of autonomic nervous activity whereas baroreflex sensitivity estimates the response of the cardiac parasympathetic system to extreme physiological stimulation. Thus both factors may be independent predictors of the post-infarction risk of arrhythmia. Currently, a large multicentre trial, ATRAMI (Autonomic Tone and Reflexes in Acute Myocardial Infarction) that involves European and North American centres, is investigating the relative and combined values of HRV and baroreflex sensitivity after myocardial infarction.

Survivors of myocardial infarction are probably the population of patients who may soon benefit most from HRV assessment. It seems that despite our limited understanding of the links between decreased HRV and increased arrhythmia risk, the time has come when depressed HRV can be used as an enrolment criterion for large clinical trials. For example, the data from large retrospective investigations suggest that it is possible to select inclusion criteria with both high sensitivity and moderate positive predictive accuracy (for example, for reducing the arrhythmia risk by $\beta$ blockade) and moderate sensitivity with high positive predictive accuracy (for example, for selecting the candidates for an implanted cardiovertor/defibrillator).

Whether therapeutic or other interventions aimed at increasing HRV in infarction survivors will also decrease their risk of arrhythmia or early mortality or both is not known. Nevertheless, many factors that are known to reduce risk, such as rehabilitation, regular training, and cessation of smoking are known to increase HRV and especially its parasympathetic components. Therapeutic interventions that increase parasympathetic tone (for example, scopolamine) may also have a positive effect on the survival of a post-infarction population with depressed HRV.

Department of Cardiological Sciences,

MAREK MALIK

Department of Cardiological Sciences,
St George's Hospital Medical School, London

A JOHN CAMM

1 Wolf MM, Varigos GA, Hunt D, Sloman JG. Sinus arrhythmia in acute myocardial infarction. Med f Austral 1978;2:52-3.

2 Kleiger RE, Miller JP, Bigger JT, et al. Decreased heart rate variability and its association with increased mortality after acute myocardia infarction. Am $\mathcal{F}$ Cardiol 1987;59:256-62.

3 Odemuyiwa O, Malik M, Farrell T, Bashir Y, Poloniecki J, Camm AJ. A comparison of the predictive characteristics of heart rate variability index and left ventricular ejection fraction for all-cause mortality, arrhythmic events and sudden death after acute myocardial infarction. Am $\mathcal{F}$ Cardiol 1991;68:434-9.

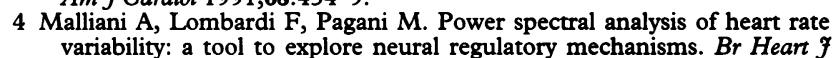
1994;71:1-2.

5 Siemens P, Hilger HH, Frowein RA. Heart rate variability and the reaction of heart rate to atropine in brain dead patients. Neurosurg Rev 1989;12:282-4.

6 Yamasaki Y, Ueda N, Kishimoto $M$, et al. Assessment of early stage autonomic nerve dysfunction in diabetic subjects-application of power nomic nerve dysfunction in diabetic subjects-application of pow
spectral analysis of heart rate variability. Diabetes Res 1991;17:73-80.

7 Krebs HB, Peters RE, Dunn LJ, Smith PJ. Intrapartum fetal heart rate monitoring. VI. Prognostic significance of accelerations. Am $\mathcal{F}$ Obstet Gynecol 1982;142:297-305.

8 Egelund N: Spectral analysis of heart rate variability as an indicator of driver fatigue. Ergometrics 1982;25:663-72.

$9 \mathrm{Ng} \mathrm{KSK}$, Gibson DG. Impairment of diastolic function by shortened filling period in severe left ventricular function. Br Heart $\mathcal{f} 1989 ; 62$ : 246-52.

10 Coumel P, Hermida JS, Wennerblom B, Leenhardt A, Maison-Blanche $P$, Cauchemez $B$. Heart rate variability in left ventricular hypertrophy and heart failure, and the effects of beta-blockade. A non-spectral analysis of heart rate variability in the frequency domain and in the time domain. Eur Heart $\mathcal{f} 1991 ; 12: 412-22$.

11 Nolan J, Flapan AD, Capewell S, MacDonald TM, Neilson JMM, Ewing DJ. Decreased cardiac parasympathetic activity in chronic heart failure

and its relation to left ventricular function. Br Heart $\mathcal{F}$ 1992;67:482-5.
Kienzle MG, Ferguson DW, Birkett CL, Myers GA, Berg WJ, Marian Kienzle MG, Ferguson DW, Birkett CL, Myers GA, Berg WJ, Mariano
DJ. Clinical, hemodynamic and sympathetic neural correlates of heart DJ. Clinical, hemodynamic and sympathetic neural correlates of heart

13 Stein KM, Borer JS, Hochreiter C, et al. Prognostic value and physiologic correlates of heart rate variability in chronic severe mitral regurgitation. Circulation 1993;88:127-35.

14 Sands KE, Appel ML, Lilly LS, Schoen FJ, Mudge GH Jr, Cohen, RJ. Power spectrum analysis of heart rate variability in human cardiac transplant recipients. Circulation 1989;79:76-82.

15 Bernardi L, Salvucci F, Saurdi $R$, et al. Evidence for an intrinsic mechanism regulating heart rate variability in the transplanted and the intact heart during submaximal dynamic exercise? Cardiovasc Res 1990;24:969-81.

16 Smith ML, Ellenbogen KA, Eckberg DL, Sheehan HM, Thames MD. Subnormal parasympathetic activity after cardiac transplantation. Am $\mathcal{f}$ Cardiol 1990;66:1243-6.

17 Guzetti S, Piccaluga E, Casati R, et al. Sympathetic predominance in essential hypertension: a study employing spectral analysis of heart rate variability. $f$ Hypertens 1988;6:711-7.

18 Casolo GC, Stroder P, Signorini C, et al. Heart rate variability during acute phase of myocardial infarction. Circulation 1992;85:2073-9.

19 Bigger JT Jr, Kleiger RE, Fleiss JL, et al. Components of heart rate variability measured during healing of acute myocardial infarction. Am $\mathcal{F}$ Cardiol 1988;61:208-15. 
20 Cripps TR, Malik M, Farrell TG, Camm AJ. Prognostic value of reduced heart rate variability after myocardial infarction: clinical evaluation of a new analysis method. Br Heart f 1991;65:14-19.

21 Algra A, Tijssen JGP, Roelandt JRTC, Pool J, Lubsen J. Heart rate variability from 24-hour electrocardiography and the 2-year risk for variability from 24-hour electrocardiograph

22 Lown B, Verrier RL. Neural activity and ventricular fibrillation. $N$ Engl $\mathcal{F}$ Med 1976;294:1165-70.

23 Hull SS Jr, Evans AR, Vanoli E, et al. Heart rate variability before and after myocardial infarction in conscious dogs at high and low risk of sudden death $7 \mathrm{Am}$ Coll Cardiol 1990;16:978-85.

24 Malik M, Farrell T, Camm AJ. Circadian rhythm of heart rate variability after acute myocardial infarction and its influence on the prognostic value of heart rate variability. Am $\mathcal{F}$ Cardiol 1990;66:1049-54

25 Bigger JT Jr, Fleiss JL, Steinman RC, Rolnitzky LM, Kleiger RE
Rottman JN. Frequency domain measures of heart period variability and mortality after myocardial infarction. Circulation 1992;85:164-73. 26 Singer $\mathrm{DH}$, Martin $\mathrm{GH}$, Magid N, et al. Low heart rate variability and sudden cardiac death. $\mathcal{F}$ Electrocardiol 1988;21 (suppl):S46-55.

27 Huikuri HV, Valkama JO, Airaksinen KE, et al. Frequency domain measures of heart rate variability before the onset of nonsustained and sustained ventricular tachycardia in patients with coronary artery sustained ventricular tachycardia
disease. Circulation 1993;87:1220-8.

28 Vybiral T, Glaeser DH, Goldberger AL, et al. Conventional heart rate variability analysis of ambulatory electrocardiographic recordings fails to predict imminent ventricular fibrillation. F Am Coll Cardiol 1993;22. $557-65$

29 Bigger JT Jr, La-Rovere MT, Steinman RC, et al. Comparison of baroreflex sensitivity and heart period variability after myocardial infarction. f Am Coll Cardiol 1989;14:1511-8.

\section{IMAGES IN CARDIOLOGY}

\section{Papillary fibroelastoma of the mitral valve: a rare cause of transient neurological deficits}

Papillary fibroelastomas are rare benign tumours of the endocardium that most commonly are found on the aortic or mitral valve. ${ }^{1}$ They are a few millimetres to some centimetres in diameter and look like sea anemones (fig). Most are found coincidentally at necropsy but a few cause patients to present with systemic emboli derived from detached fronds of tumour or from thrombi developing between the fronds.

This specimen $(2.3 \mathrm{~cm}$ in diameter) was removed from the mitral sub-valve apparatus of a man of 59 with a history of two transient cerebral ischaemic attacks. The mitral valve was replaced. Routine echocardiography showed an intracavity mass within the left ventricle. Since the operation a year ago he has had no further cerebral ischaemic attacks.

Papillary fibroelastomas are different from myxomas. Not every intracavitary mass is a myxoma. If a fibroelastoma is recognised it can simply be peeled away from the underlying tissue and the valve can be preserved.

J MANN

D J PARKER

1 McAllister HA, Fenoglio JJ. Tumours of the cardiovascular system. Armed Forces Institute of Pathology 1978:20-1. 